\title{
Application of 3D Printed Biocompatible Plastic Surgical Template for the Reconstruction of a Nasoalveolar Cleft with Preoperative Volume Analysis
}

\author{
ZOLTAN FABIAN ${ }^{1 *}$, KRISTOF KADAR ${ }^{2,3}$, LAJ OS PATONAY3 $^{3}$ KRISZTIAN NAGY 4 \\ ${ }^{1}$ UMFST Targu Mures \\ ${ }^{2}$ Department of Oral Biology, Faculty of Dentistry Semmelweis University, Budapest \\ ${ }^{3}$ Kreativ Dental Clinic, Budapest \\ 4 stDepartment of Pediatrics, Semmelweis University, Budapest
}

\begin{abstract}
Secondary autogenous bone grafting is the gold standard for the closure of an alveolar cleft. A preoperative surgical planning helps to determine the optimal shape and volume of the graft, that ideally guide canine eruption to the cleft area and ensure adequate nasal support. The authors describe their experience with design and use of an individually shaped 3D template for an autologous bone graft with fibrin glue for correct volume design. The conforming template is designed using freeware and open source software, it is manufactured by means of additive technology using autoclavable resin.
\end{abstract}

Keywords: alveolar cleft, 3D reconstruction, surgical template, secondary alveolar bone graft

Secondary autogenous bone grafting was introduced in the 1980's and became the method of choice for the repair of the nasoalveolar defectin cleft patients. Since then, there has been a continuous research to ensure favorable results [1]. The alveolar cleft is a complex three-dimensional (3D) midfacial soft tissue and bony deformity and surgical management benefits from adequate planning. Accurate evaluations of the cleft dimensions became possible with the rising of 3D imaging technology [2,3]. The preoperative definition of the architecture and size of the bony defect has been considered helpful because those features may vary with the cleft type and show great variability between patients. Radiographic 3D imaging is considered state of the artfor the diagnostic evaluation and treatment planning of patients requiring alveolar bone grafts [4]. Studies have already showed that software simulations, based on 3D cone beam CT data, are reliable and reproducible, thus can be implemented for the planning of cleft surgery [4].

Palhazi et al. described the use of a graft template to ensure adequate grafting of the alveolar cleftand adequate nasal support [2]. CBCT (cone beam computed tomography) data acquisition was performed in order to visualize the alveolar cleft. An adequate estimation was performed to determine the volume of the bone graft. The iPlan surgical planning software (Brainlab AG, Feldkirchen, Germany) was used to create $3 \mathrm{D}$ virtual template of the naso-alveolar cleft. Previously, this template was used as a guide for trimming the bone graft to the proper shape of the cleft in the operating room, however, this method was found inaccurate and time-consuming.

Our main objective was to develop a workflow to create a custom-made, individualized surgical template for provide help in harvesting adequate autologous bone and to conform its shape to the alveolar bone defect for each patient.

\section{Experimental part}

Materials and methods

We present an inexpensive and straightforward workflow for creating a patient specific 3D printed resin mold for obtaining a perfectly fitting bony cast of the alveolar defect, using autologous cancellous bone from the hip, mixed with fibrin adhesive (Tissucol, BAXTER). We also present our short-term experience in using these devices.

The preoperative CBCT data of the alveolar defect was used obtained to create the 3D model of CT images was also used for the evaluate the upper airways, asymmetries and developmental of the facial skeleton.

The 3D model of the bony defect was created using Osirix (Pixmeo SARL) by the following method:

The region of interest (ROI) of the bony defect was marked on each slice by contouring the borders of the defect using the ROI pencil tool. Adequate precision and a significant reduction in worktime can be achieved by manually contouring only a fraction of the slices, while using the software's interpolation function to calculate the inbetween slices. The tracing of the defect was carried out using a graphic tablet (Intuos Pro, WACOM) for a convenient and precise contour drawing. The volume of interest (VOI) of the defect was calculated by the software from the ROI set. The Vol was transformed to a mask by using Set pixel values to ... function (ROI menu). The mask was further converted to a mesh model by segmentation and surface rendering.

The mesh model of the alveolar cleft was exported as an STL (standard tessellation language) file into Meshmixer software (Autodesk). The Smooth tool was used to carry out a shape preserving smoothing of the sharp edges. The resulted mesh may serve after 3D printing as a visual aid during bone graft collection from the hip. However, we took the planning a step further, by subtracting the 3D model of the defect from a solid, obtaining this way the negative of the defect, which can be 
used as a casting mold for the conformation of the particulate bone. To facilitate the cast removal, a two-part mold with an interlocking mechanism was created and the upper face of the mesh was selectively extruded and subtracted from the mold, thus obtaining an undercut removal. As a final step a cover was created from cylindrical primitives using the software's boolean substraction function to conform the morphology of the upper surface of the graft.

The cast is made out of three parts. These parts were 3D printed using an OBJET 500 (Stratasys, Israel) using a clear MED610 (Stratasys, Israel) biocompatible resin. The support material was made from SUP705 (Stratasys, Israel) resin. MED610 is a resin made of exo-1,7,7-trimethylbicyclo [2.2.1] hept-2-yl acrylate, 4-(1-oxo-2-propenyl)-morpholine, 2Propenoic acid, Glycerol, propoxylated, esters with acrylic acid and some proprietary, undisclosed components. This biocompatible resin, is compliant with ISO 10993-1 with a Category 3 specific target organ toxicity. The materials tensile strength is $50-65 \mathrm{MPa}$, its elasticity modulus is $2000-3000 \mathrm{MPa}$.

The support material is a waterjet removable resin, made from the following components: poly(oxy-1,2ethanediyl), alpha-(1-oxo-2-propenyl)-omega-hydroxy-, 1,2-Propylene glycol, polyethylene glycol, glycerin, phosphine oxide, phenylbis(2,4,6-trimethylbenzoyl)-, acrylic acid ester.

The used 3D printer is a multi-color, multi-material printer, using inkjet printing technique and UV curing for printing high quality parts.

\section{Results and discussions}

The planning and manufacturing were straightforward. Our parts were printed with a layer thickness of 16 microns. We used these medical devices in 10 cases of secondary cleft surgery ( 8 unilateral, 2 bilateral). The steam sterilization process did not alter the dimensions and transparency of the device. The surgical interventions were carried out, there were no early complications.

Additional benefits are the possibility for compacting the bony particles before inserting into the surgical field.

The surfaces that are in contact with the mucosa or periosteum can be marked and a
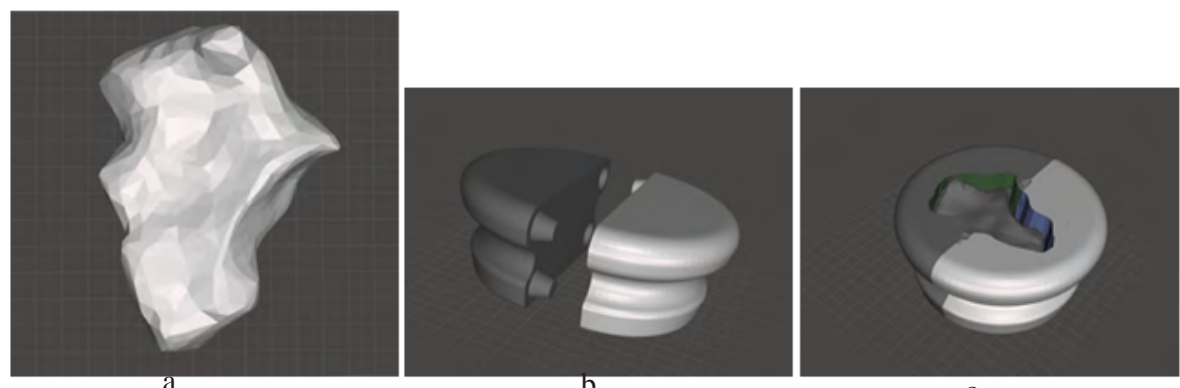

Fig. 1. 3D model of the graft (a), of the tray model (b), and tray model with the hollow and the graft inside (c)
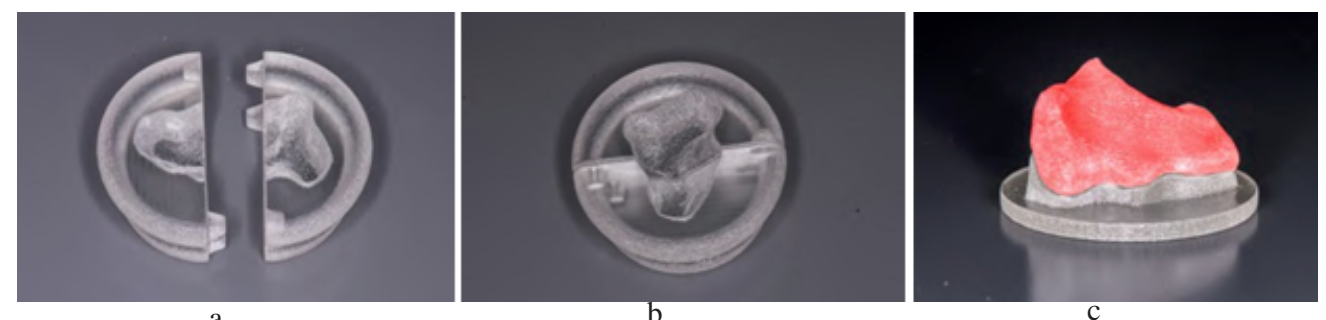

Fig. 2. Two part mold with the interlocking mechanism separated (a) and assembled (b). Cover and the graft mockup (c) 
anatomical characteristics of cleft [2]. This template can also be used to create a 3D mold for the transfer for the actual amount of bone that is required, instead of the syringe compression method (3). Every patient is individually different, and the $3 \mathrm{D}$ CB-CT imaging helps to estimate the location and degree of the maxillary deformity [5]. Three-dimensional images showed very precise results for volumetrically analysis of bone graft volumes $[1,3,4]$. Now it also important to translate these accurate measurements into practical surgical skills. Only with accurate volume of bone grafting we can ensure optimal clinical outcome and correct values for study evaluations. By implementing this technique, we can obtain correct study data to evaluate bone resorption and to truly understand the bone regeneration in the 3D anatomical naso-alveolar cleft defect. Our technique ensures a correct and meticulous preparation of the surgical case, ensures less surgical trauma and a short operation time. Accurate evaluation of the CBCT data gives a correct evaluation of the alveolar cleft volume and the amount of bone that is required for grafting.

\section{Conclusions}

Our workflow is straightforward, using freeware software for planning.

The MED610 biocompatible material is a good choice for $3 \mathrm{~d}$ printing this medical device. This method brings an added value to the secondary cleft surgery, by increasing the bone-graft volumetric precision and its density, while decreasing the duration of the surgery. We intend to further study the impact of this method on dynamics of the bone graft on long term, by follow up study for assessing and comparing the resorption ratio of these grafts versus the resorption after the classic grafting procedure.
Acknowledgement: The work presented in this article was partly funded by the KFI_16-1-2017-0004 research project.

\section{References}

1.FEICHTINGER M, ZEMANN W, MOSSBOCK R, KARCHER $H$. Threedimensional evaluation of secondary alveolar bone grafting using a 3D- navigation system based on computed tomography: a two-year follow-up. Br J Oral Maxillofac Surg. 2008;46(4):278-82.

2.PALHAZI P, NEMES B, SWENNEN G, NAGY K. Three-dimensional simulation of the nasoalveolar cleft defect. Cleft Palate Craniofac J. 2014;51(5):593-6.

3.ZHOU WN, XU YB, JIANG HB, WAN L, DU YF. Accurate Evaluation of Cone-Beam Computed Tomography to Volumetrically Assess Bone Grafting in Alveolar Cleft Patients. J Craniofac Surg. 2015;26(6):e535-9. 4.OH TS, PARK JS, CHOI JW, KW ON SM, KOH KS. Risk factor analysis of bone resorption following secondary alveolar bone grafting using three-dimensional computed tomography. J Plast Reconstr Aesthet Surg. 2016;69(4):487-92.

5.SHIROTA T, KURABAYASHI H, OGURA H, SEKI K, MAKI K, SHINTANI $\mathrm{S}$. Analysis of bone volume using computer simulation system for secondary bone graft in alveolar cleft. Int J Oral Maxillofac Surg. 2010;39(9):904-8.

6. SEGURA-CASTILLO JL, AGUIRRE-CAMACHO H, GONZÁLEZ-OJEDA A, MICHEL-PEREZ J. Reduction of bone resorption by the application of fibrin glue in the reconstruction of the alveolar cleft. J Craniofac Surg. 2005J an;16(1):105-12.

7. GUO J, LI C, ZHANG Q, WU G, DEACON SA, CHEN J, HU H, ZOU S, YE Q. Secondary bone grafting for alveolar cleft in children with cleft lip or cleft lip and palate. Cochrane Database Syst Rev. 2011 J un 15;(6):CD008050. doi:10.1002/14651858.CD008050.pub2.

Manuscript received: 19.12 .2018 